\title{
The effect of restriction membranes on mass transfer in an electrodialysis with filtration membrane process
}

\author{
Huining Denga, George Q.Chen ${ }^{b}$, Sally L. Gras ${ }^{b, c}$, Sandra E. Kentish ${ }^{b, *}$ \\ a School of Marine Science and Engineering, Hebei University of Technology, Tianjin 300130, P. R. China \\ b Department of Chemical and Biomolecular Engineering, The University of Melbourne, Victoria 3010, \\ Australia
}

cBio21 Molecular Science and Biotechnology Institute, The University of Melbourne, Victoria 3010, Australia

\begin{abstract}
In an electrodialysis with filtration membrane (EDFM) separation process, a pair of restriction membranes is used to prevent leakage of molecules into the electrode solution. Driven by the electrical potential gradient, ions transport across these membranes in different directions, resulting in concentration changes in the feed and permeate compartments respectively. However, the effects of these restriction membranes have not been systemically studied to date. In the present work, the performance of a non-charged polyacrylamide (PAm) membrane and two ion-exchange membranes were compared. PAm restriction membranes were found to give the most stable operation, with only slight concentration and $\mathrm{pH}$ changes in the feed and permeate compartments. The configurations with either cation or anion exchange membranes showed more significant changes in electrolyte concentration in opposing directions but the same $\mathrm{pH}$ trend, as the current density increased. The configuration with cation exchange membranes as restriction membranes resulted in the highest protein recovery, while the use of anion exchange membranes would appear as the best choice for separation of a protein with net positive charge. The results of this work provide important insights for the design and operation of EDFM processes and a better understanding of the transport phenomena that occur during the separation of bioactive compounds.
\end{abstract}

Keywords: Electrophoresis; electrodialysis, protein separation; ion exchange membranes.

*Corresponding author.Tel.: +61383446682; Fax: +6183444153. E-mail address: sandraek@unimelb.edu.au (S.E. Kentish). 


\section{Introduction}

Electrodialysis with filtration membrane (EDFM) is an emerging membrane technology for fractionation of electrically charged bioactive compounds such as proteins or their peptides $[1,2]$. Driven by an electric potential difference across the filtration membrane, the EDFM process shows improved selectivity and reduced fouling compared to pressure-driven membrane processes $[3,4]$. Selective transport of the bioactive molecules across the separation membrane is achieved by both differences in charge and molecular size. As the approach is similar to electrophoresis, it is sometimes referred to as an electrophoretic membrane contactor, particularly if a larger porous membrane is used as the separation medium for protein separation [5-8]. One or a series of separation membranes with different pore sizes has been used to extract proteins from egg white [9] and whey [7], to extract peptides from protein hydrolysate $[10,11,12]$, and to purify charged molecules with low molecular weight [13]. Bazinet and co-workers have studied the effect of the electric field [1416], $\mathrm{pH}$ [17], ionic strength [4] and membrane physicochemical properties [18] on both the separation ability and membrane fouling within the EDFM process; but an understanding and optimization of the EDFM process is far from complete.

A basic EDFM configuration is composed of one separation membrane, two restriction membranes and a pair of electrodes, as shown in Fig. 1. Normally, buffers are used as the cathode and anode solutions and are circulated in a closed loop to neutralize the base generated from cathode reactions, with acid from the anode. The two inner compartments can be designated to be feed and permeate, these are allocated according to the direction of the electric field and the charge of the protein to be extracted. The separation membrane is selected based on the charge and molecular weight of the mixture to be fractionated. Since the characteristics of this membrane determine the selectivity and flux of EDFM, the 
membrane charge, apparent pore radius and electro-osmotic flux have been intensively studied $[6,13,19]$. However, few works have focused on the effect of the restriction membranes on the EDMF process. The restriction membranes act to conduct electric current and restrict molecules from leaking into the electrode compartments. Their charge and pore size will also influence the transport of ions in the EDFM and are therefore an important consideration.

Gel membranes are commonly used as restriction membranes $[9,20]$, where the current is carried by the ions in the electrolyte solution which swells the membrane structure. Neutral materials such as polyacrylamide (PAm) are typically selected for this purpose. The restriction membranes have a tighter structure than separation membranes, which is adjusted by the degree of crosslinking (see Fig. 1 (a)). Suitable restriction membranes normally have a molecular weight cut-off just smaller than the molecular weight of the smallest proteins in the mixture to be separated.

Ion-exchange membranes (IEMs) offer an alternative for restriction membranes. They exhibit low resistance to counter-ion migration because of their high charge density. Moreover, the migration of molecules with molecular weight exceeding 500 Dalton is prohibited due to their dense structure [21-23]. Both cation exchange membranes (CEM) and anion exchange membranes (AEM) can be used [22, 23], in four different configurations, as shown in Fig. 1. These are two CEMs (Fig. 1 (b)), two AEMs (Fig. 1 (c)), AEM and CEM with CEM adjoining the cathode (Fig. 1 (d)), or AEM and CEM with CEM adjoining the anode (Fig. 1 (e)). 


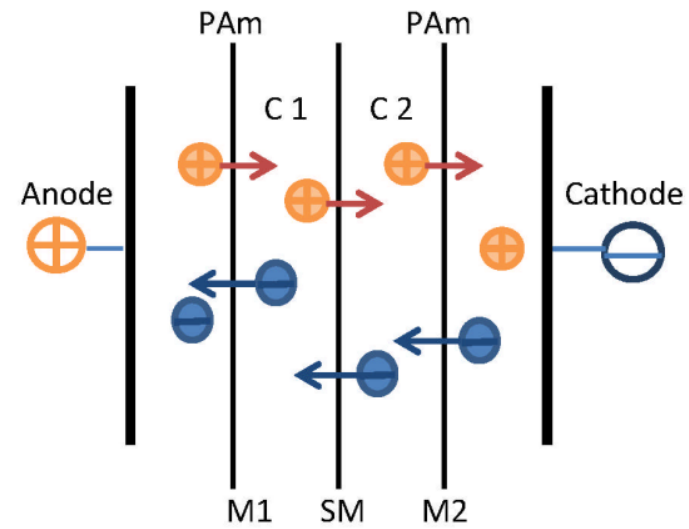

(a)

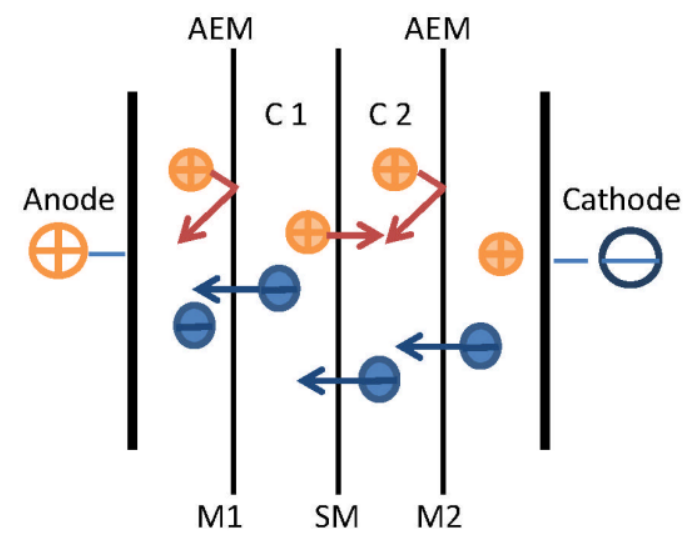

(c)

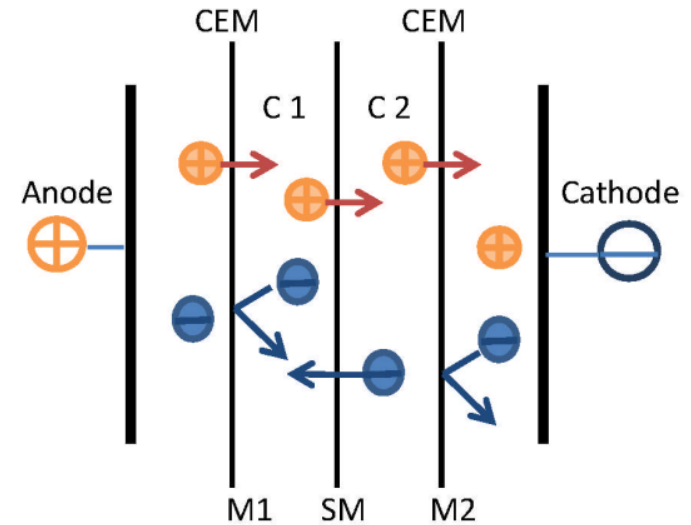

(b)

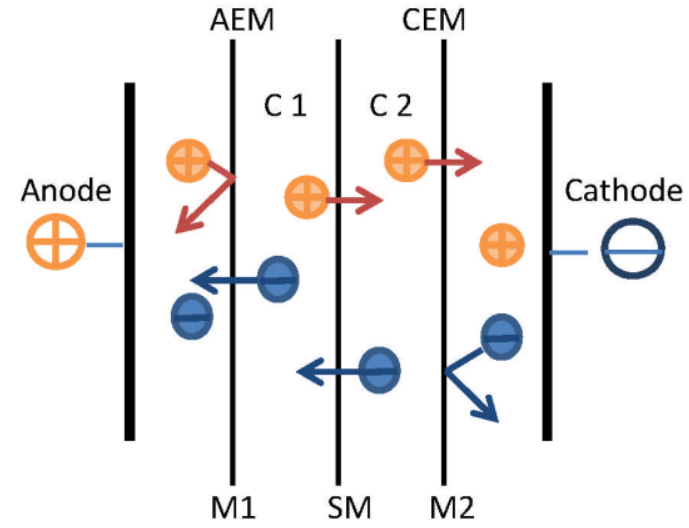

(d)

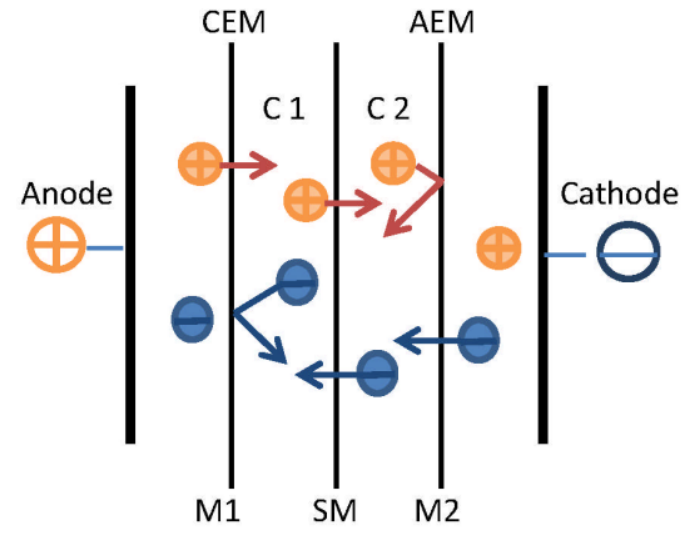

(e)

Fig. 1. EDFM configurations with ion-exchange membranes (cation-exchange membranes, CEM or anion-exchange membranes, $A E M)$ or non-charged polyacrylamide (PAm) membranes acting as restriction membranes. SM is the separation membrane, M1 and M2 are the two restriction membranes and $\mathrm{C} 1$ and $\mathrm{C} 2$ are the two inner compartments used for the feed and permeate solutions. The movement of positively and negatively charged species is indicated by the arrows. 
In the configuration shown in Fig. 1(d), cations and anions in the two inner compartments flow out to the electrode compartment through the CEM and AEM respectively, driven by the electric potential gradient. This will continuously increase the concentration of ions in the electrode buffer. Conversely, the ions in the electrode buffer solution will flow into the inner feed and permeate compartments in the configuration shown in Fig. 1 (e). Even in a continuous process with constant feed and permeate inlet composition, this would cause the composition of the electrode buffer to change and result in a change in current density. Consequently, these two configurations will be unstable and are unlikely to be used for any practical applications. Therefore, in this study we focus on the first three configurations (Fig. $1(\mathrm{a}-\mathrm{c}))$, in which stable operation can be realized.

This study focuses on investigating the effect of these three stable configurations on mass transfer within an EDFM operation, both experimentally and using a theoretical approach. The change in ion concentration and $\mathrm{pH}$ in both the feed and permeate compartment, together with the protein transfer flux are studied using bovine serum albumin (BSA) as a model protein and a non-charged polyacrylamide (PAm) membrane as the separation membrane.

\section{Theoretical background}

In an EDFM system consisting of three membranes and a pair of electrodes, the change in concentration in each compartment is determined by ion transport through the three membranes. In an electrical potential driven process, the flux of cations and anion are interdependent because of the requirement for electroneutrality in the solutions. The change in concentration in compartment $1\left(\Delta C_{1}\right)$ and compartment $2\left(\Delta C_{2}\right)$ can be calculated by the 
sum of the anion or cation flux $(\mathrm{J})$ in and out of the compartments. With cation transfer for example, the concentration changes in compartment 1 and 2, as shown in Fig. 1, are

$$
\begin{gathered}
\Delta C_{1}=z_{C} J_{C M 1}-z_{C} J_{C S M} \\
\Delta C_{2}=z_{C} J_{C S M}-z_{C} J_{C M 2}
\end{gathered}
$$

where $\mathrm{z}$ is the valence, $\mathrm{J}$ is the flux and the subscripts $\mathrm{M} 1, \mathrm{M} 2$ and SM refer to the restriction membrane next to the anode, next to the cathode and the separation membrane respectively, as shown in Fig. 1. The subscript $\mathrm{C}$ refers to cations.

It is assumed that the current through the membranes is carried by ions only, i.e.,

$$
I=F \sum_{i} z_{i} J_{i}=F\left(z_{C} J_{C}+z_{A} J_{A}\right)
$$

where $\mathrm{I}$ is the current density, $\mathrm{A} / \mathrm{cm}^{2}, \mathrm{~F}$ the Faraday constant, and the subscript I and $\mathrm{A}$ refers to each ionic species, and anions, respectively. For protein separation, a negatively charged protein is included as an anion and a positively charged protein as a cation.

Introducing Equation 3 to Equations 1 and 2 leads to:

$$
\begin{aligned}
& \frac{\Delta C_{1}}{I / F}=\frac{z_{C} J_{C M 1}}{z_{C} J_{C M 1}+z_{A} J_{A M 1}}-\frac{z_{C} J_{C S M}}{z_{C} J_{C S M}+z_{A} J_{A S M}} \\
& \frac{\Delta C_{2}}{I / F}=\frac{z_{C} J_{C S M}}{z_{C S M} J_{C}+z_{A} J_{A S M}}-\frac{z_{C} J_{C M 2}}{z_{C} J_{C M 2}+z_{A} J_{A M 2}}
\end{aligned}
$$

The ion transport number, $T_{i}$, is defined by the fraction of the current that is carried by a certain ion, i.e.,

$$
T_{i}=\frac{z_{i} J_{i}}{\sum_{i} z_{i} J_{i}}
$$

Then, the concentration change in compartment 1 is

$$
\frac{\Delta C_{1}}{I / F}=T_{C M 1}-T_{C S M}
$$

Similarly, the concentration change in compartment 2 is 


$$
\frac{\Delta C_{2}}{I / F}=T_{C S M}-T_{C M 2}
$$

From Equations 7 and 8, the concentration change in the compartments is proportional to the current density and the transport number difference between the two adjacent membranes. The transport number of each membrane, i.e. its charge selectivity is determined by the concentration of co-ions and counter-ions in the membrane, which again depends on the ion-exchange capacity of the membranes.

For the EDFM process, most attention is paid to the protein transport flux. The anion transport number is fixed for a given separation membrane, which means the total flux of the negatively charged ions and protein only depends upon the current. Taking the negatively charged BSA as an example, the negative charge transfer across the separation membrane is caused by the sum of flux of anions and the negatively charged BSA, i.e.,

$$
z_{A} J_{A S M}=z_{A} J_{A^{\prime} S M}+z_{B S A} J_{B A S S M}
$$

where subscript $A^{\prime}$ refers to all anions except the proteins.

The fluxes of these smaller anions can be expressed by the Nernst-Plank Equation[24] as defined in Equation 10:

$$
J_{A^{\prime}}=-D_{A^{\prime}} \frac{d C_{A^{\prime}}}{d x}-D_{A^{\prime}} \frac{Z_{A^{\prime}} C_{A^{\prime}} F}{R T} \frac{d \varphi}{d x}
$$

where $D_{A^{\prime}}$ is the Fickian diffusion coefficient of the anions $A^{\prime}$ in the membrane, $C_{A^{\prime}}$ is the anion concentration in the membrane and $\varphi$ is the electrical potential across the membrane. The permeate flux of ions is determined by two components i.e. ion diffusion and migration driven by the electrical potential gradient. The permeability of negatively charged protein can be calculated by subtracting the anion flux from the total negatively charged flux with Equation 9. Together these equations provide a useful description of the EDFM process. 


\section{Experimental}

\subsection{Materials}

Bovine serum albumin (BSA) was purchased from Sigma Aldrich, Castle Hill, NSW, Australia. The polyacrylamide (PAm) gel membranes with molecular weight cut-off (MWCO) of $5000 \mathrm{Da}$ and 250,000Da used as restriction membrane and separation membrane respectively were kindly provided by Memphasys Limited, Homebush West, NSW, Australia. The Tris-base and Hepes for buffer preparation were acquired from Sigma Aldrich (Castle Hill, NSW, Australia) and Formedium (Hunstanton, Norfolk, United Kingdom), respectively. The cation exchange membranes (Neosepta $\mathrm{CMX}$ ) and anion exchange membranes (Neosepta AMX) were purchased from Astom Corporation, Minato-ku, Tokyo, Japan. All the membranes were immersed in the buffer solution for more than $24 \mathrm{~h}$ before use.

The choice of buffer is a major factor for ensuring the separation performance in the EDFM process and buffers of low conductivity are recommended [9, 25]. A Tris-Hepes buffer prepared with $20 \mathrm{mM}$ of Tris-base, $10 \mathrm{mM}$ of Hepes and $1 \mathrm{mM}$ of Ethylenediaminetetraacetic acid disodium salt $\left(\mathrm{EDTANa}_{2}\right)$ at $\mathrm{pH}$ of 8.00 was used in both the separation and the electrode compartments. This solution has both the cation and the anion buffer at the desired $\mathrm{pH}$, improving the buffering capacity without increasing in the ionic strength and is widely used in studies on bioactive compounds [26-28].The feed solution with a protein concentration of $1 \mathrm{~g} / \mathrm{L}$ was prepared by dissolving the appropriate amount of protein in this buffer. The adjustment of $\mathrm{pH}$ with inorganic acid or base was avoided to maintain the low ionic strength required.

\subsection{Experimental procedure and operating conditions}


The EDFM test was performed on a BF400 assembly kindly provided by Memphasys Limited, Sydney, Australia, which is composed of one separation membrane, two restriction membranes and a pair of electrodes, as shown in Fig. 1. Two plastic spacers are inserted between the separation and restriction membranes to form the feed and permeate compartments. BSA is negatively charged in the Tris-Hepes buffer, so the compartment adjacent to the cathode (compartment 2, as shown in Fig. 1(a-e)) is the feed and the other is the permeate. A PAm gel membrane with an active area of $15.6 \mathrm{~cm}^{2}$ was used as the separation membrane. This non-charged membrane is selected to minimize the permeation of water caused by electro-osmosis. PAm membranes with MWCO of $5000 \mathrm{Da}$ and commercial ion-exchange membranes were used as restriction membranes according to the configurations shown in Fig. 1(a-c). The electrode buffer was recirculated via a tank placed inside the BF 400 and chilled with iced water to maintain solution temperatures below $15^{\circ} \mathrm{C}$. During continuous processing, the feed and permeate solutions were directed to flow in a single pass through the membrane module with a flow rate of $16 \mathrm{~mL} / \mathrm{min}$ at a constant voltage, within the range of 50 to $210 \mathrm{~V}$. The solutions from the feed and permeate were collected separately after three minutes of operation and their conductivity, $\mathrm{pH}$ and protein concentrations determined. Following this, the electricity was disconnected and the flows diverted to a waste container to refresh the compartments with fresh solution. A new voltage was then initiated and after around $30 \mathrm{sec}$ the flows were directed back to the feed and permeate collection vessels.

For batch operation, $100 \mathrm{~mL}$ of solution was used for both feed and permeate, with each circulated separately in a closed loop at a flow rate of $16 \mathrm{~mL} / \mathrm{min}$. The conductivity and $\mathrm{pH}$ of both solutions were monitored continuously and the protein concentration was determined before and after the 25 -min operation. The batch operations were performed at a constant 
voltage of $100 \mathrm{~V}$. The conductivity and $\mathrm{pH}$ of the electrode buffer were monitored before and after the experiment.

The protein transfer was estimated with the following equation:

$$
\text { Transfer }=\frac{m_{p} C_{p}}{m_{f} C_{f}+m_{p} C_{p}} \times 100 \%
$$

Where $m_{f}$ and $m_{p}$ are the mass of feed and permeate solution, respectively, and $C_{f}$ and $C_{p}$ are the concentration of feed and permeate solution.

The conductivity and $\mathrm{pH}$ of the solutions were monitored by a $\mathrm{pH}$ meter (HannaHI251) and conductivity meter (Crison Basic 30+). The concentration of BSA was determined with a UV/vis spectrophotometer (Agilent CARY 300) at $280 \mathrm{~nm}$.

\section{Results and discussion}

\subsection{Changes in conductivity and $\mathrm{pH}$}

For electrically driven processes such as EDFM, the current is carried by ion migration between the different compartments across the membrane. The concentration changes resulting from this ion migration were monitored by conductivity. The two-PAm and two-CEM systems exhibit a linear conductivity increase in $\mathrm{C} 1$ and a corresponding decrease in $\mathrm{C} 2$ as the operating current increases during continuous processing (Fig. 2(a)). These results indicate a linear change in electrolyte concentration with the increase of current density. The system with CEMs as restriction membranes shows a significantly greater change in conductivity compared to that using PAm membranes. Conversely, the system with two AEMs as restriction membranes shows a different trend (Fig. 2 (b)). The conductivity of the feed solution in C1 exhibits an obvious downward trend, but the conductivity in C2 increases slightly above the feed value at low current density and then gently declines with further 
current increases. It should be noted that the $\mathrm{pH}$ and conductivity of the electrode buffer did not show any variation before and after the experiment with the three different configurations.
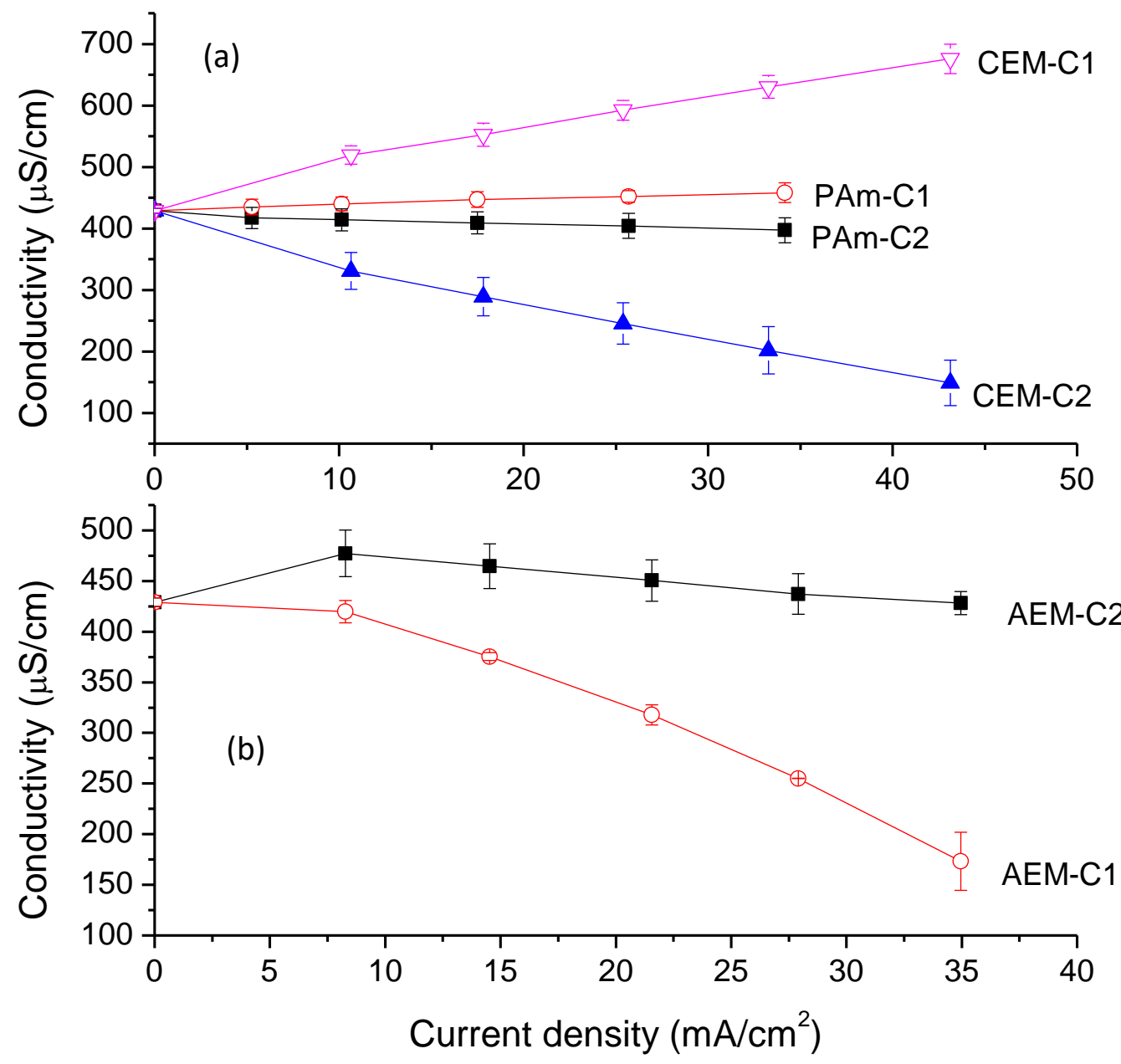

Fig. 2.Conductivity change in compartment 1 and 2 as a function of current density during continuous processing in (a) configurations with PAm and CEM as a restriction membrane and (b) a configuration with AEM as a restriction membrane. The voltage varies from 0 to $210 \mathrm{~V}$. Error bars are based on replicate experiments.

The conductivity changes for the PAm and CEM configurations can be explained by Equations 7 and 8 , where the concentration change in the compartments is proportional to the current and the difference in transport number between the two adjacent membranes. In a 
membrane stack, such as this EDFM system, the current density across every membrane has the same value. The cation transport number of each membrane, however, is quite different, which results in a different concentration change. In IEMs, the co-ions are excluded due to the fixed charged groups in the membrane matrix. If IEMs are assumed to act as strictly permselective membranes, the transport number of the counter-ion can be regarded as 1 . Therefore, to take the two-CEM configuration as an example, the change of concentration $\Delta C_{1}$ is

$$
\frac{\Delta C_{1}}{I / F}=1-T_{C S M}=T_{A S M}
$$

Similarly, $\Delta C_{2}$ can be expressed as:

$$
\frac{\Delta C_{2}}{I / F}=-T_{A S M}
$$

Normally, the transport number of the porous separation membrane is somewhere between 0 and 1 . Therefore, as the concentration of $\mathrm{C} 1$ increases and $\mathrm{C} 2$ decreases, there should be a net ion transfer from $\mathrm{C} 2$ to $\mathrm{C} 1$. Similar conductivity changes were also observed by Chen et al. in electro-ultrofiltration operation with two CEMs[29]. This direction of ion transfer will be the same for all kinds of separation membranes, although the actual values of ion permeability may differ due to the inherent selectivity of the separation membrane. Specifically, if a CEM was used as the separation membrane (i.e. $T_{A S M}=0$ ), the flux of cations into the compartments will equal that flowing out and there will be no concentration change in either compartment. If the separation membrane is an AEM, then the two adjacent compartments become simultaneously concentrated and diluted as in the standard electrodialysis configuration, which has the highest salt concentrating efficiency.

The calculated concentration changes in the other configurations, shown in Fig. 1, are provided in Table 1. The concentration in the electrode compartment will change in an inverse 
manner to the sum of the two inner compartments $\left(\frac{\Delta C_{1}}{I / F}+\frac{\Delta C_{2}}{I / F}\right)$ through consideration of the mass balance. On this basis, the electrode compartment will be stable in composition when operating at any current for the configurations shown in Figs. 1 (a), (b) and (c). The configurations with a pair of CEMs and AEMs should show a net flow out (Fig. 1 (d)) or flow in (Fig. $1(\mathrm{e})$ ) to the two inner compartments, which has been already demonstrated by experiments in the literature $[12,18,30]$. The conductivity variation with the two PAm restriction membranes shown in Fig. 2 suggests that the cation transport number of the restriction membrane is slightly higher than that of the separation membrane $\left(T_{C M 1}>T_{C S M}\right)$. This may be caused by different charge densities within the membrane structures, related to differing degrees of crosslinking.

The configuration with two AEMs should exhibit concentration changes in the feed and permeate in an opposite direction to that of the two-CEM system. However, this is not in accordance with the experimental results shown in Fig. 2 (b). 
Table 1. Calculated concentration change for each of the different configurations as shown in Fig. 1.

\begin{tabular}{|c|c|c|c|c|c|c|c|}
\hline Configuration & $\begin{array}{l}\text { Rest } \\
\text { men }\end{array}$ & $\begin{array}{l}\text { ction } \\
\text { rane }\end{array}$ & $\mathrm{T}_{\mathrm{A}}$ & $\mathrm{T}_{\mathrm{c}}$ & $\frac{\Delta C_{1}}{I / F}$ & $\frac{\Delta C_{2}}{I / F}$ & $\frac{\Delta C_{1}}{I / F}+\frac{\Delta C_{2}}{I / F}$ \\
\hline \multirow{2}{*}{ Fig. 1 (a) } & M1 & PAm & $0 \sim 1$ & $0 \sim 1$ & \multirow{2}{*}{$\mathrm{T}_{\mathrm{CM} 1}-\mathrm{T}_{\mathrm{CSM}}$} & \multirow{2}{*}{$\mathrm{T}_{\mathrm{CSM}}-\mathrm{T}_{\mathrm{CM} 2}$} & \multirow{2}{*}{0} \\
\hline & M2 & PAm & $0 \sim 1$ & $0 \sim 1$ & & & \\
\hline \multirow{2}{*}{ Fig. 1 (b) } & M1 & CEM & 0 & 1 & \multirow{2}{*}{$\mathrm{T}_{\mathrm{ASM}}$} & \multirow{2}{*}{$-T_{A S M}$} & \multirow{2}{*}{0} \\
\hline & M2 & CEM & 0 & 1 & & & \\
\hline \multirow{2}{*}{ Fig. 1 (c) } & M1 & AEM & 1 & 0 & \multirow{2}{*}{$-\mathrm{T}_{\mathrm{CSM}}$} & \multirow{2}{*}{$\mathrm{T}_{\mathrm{CSM}}$} & \multirow{2}{*}{0} \\
\hline & M2 & AEM & 1 & 0 & & & \\
\hline \multirow{2}{*}{ Fig. 1 (d) } & M1 & CEM & 0 & 1 & \multirow{2}{*}{$\mathrm{T}_{\mathrm{ASM}}$} & \multirow{2}{*}{$\mathrm{T}_{\mathrm{CSM}}$} & \multirow{2}{*}{$\mathrm{T}_{\mathrm{ASM}}+\mathrm{T}_{\mathrm{CSM}}$} \\
\hline & M2 & AEM & 1 & 0 & & & \\
\hline \multirow{2}{*}{ Fig. 1 (e) } & M1 & AEM & 1 & 0 & \multirow{2}{*}{$-T_{C S M}$} & \multirow{2}{*}{$-T_{A S M}$} & \multirow{2}{*}{$-\mathrm{T}_{\mathrm{CSM}}-\mathrm{T}_{\mathrm{ASM}}$} \\
\hline & M2 & CEM & 0 & 1 & & & \\
\hline
\end{tabular}

To investigate the reason why the experimentally determined conductivity for the two-AEM system deviated from that shown in Table 1, the $\mathrm{pH}$ changes in the three stable configurations were compared during continuous processing. As shown in Fig. 3, the $\mathrm{pH}$ was found to change in the same direction for two-AEM and two-CEM restriction membrane configurations during continuous processing, i.e., the $\mathrm{pH}$ of the solution in $\mathrm{C} 1$ decreased while that in the $\mathrm{C} 2$ increased with the current density. This is contrary to the conductivity results for these systems. Conversely, the $\mathrm{pH}$ values of the solutions in $\mathrm{C} 1$ and $\mathrm{C} 2$ are relatively stable in the configuration with non-charged PAm restriction membranes. 


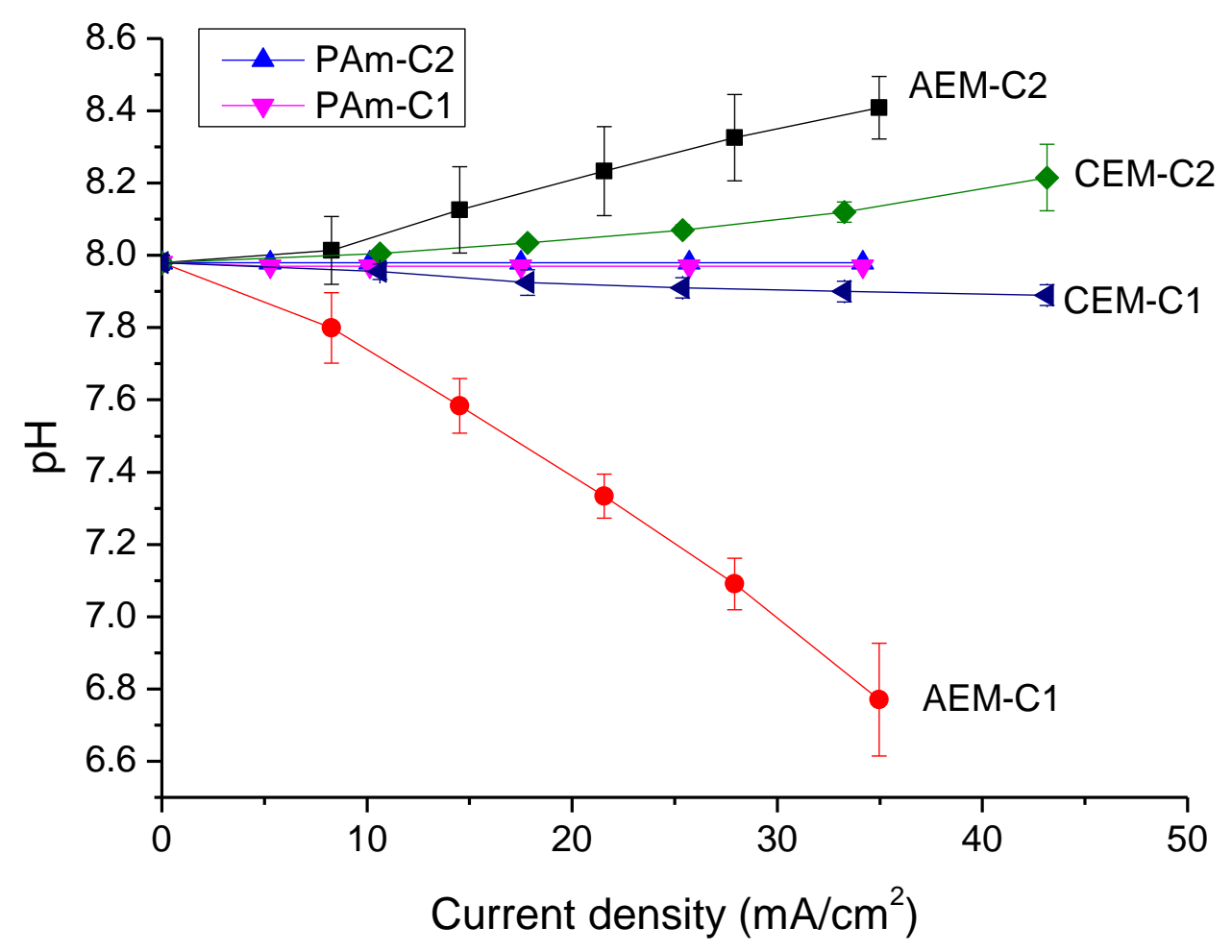

Fig. 3.Measured $\mathrm{pH}$ changes in the compartment 1 and 2 as a function of current in continuous processing with different configurations. The voltage varies from 0 to $210 \mathrm{~V}$. Error bars are based on replicate experiments.

The main source of ions in the system arises from the Tris-Hepes buffer, which consists of a weak base and a weak acid in combination (Fig. 4). Both will be partially dissociated with the percentage dissociation affected by the $\mathrm{pH}$ value according to Equation 14 and 15 :

$$
\begin{array}{lr}
\text { Tris } H^{+} \rightleftharpoons H^{+}+\text {Tris } & p K a=8.07 \\
\text { Hepes } \rightleftharpoons \text { Hepes }^{-}+H^{+} & p K a=7.5
\end{array}
$$


<smiles>NC(CO)(CO)CO</smiles>

Tris-base<smiles>O=S(=O)(O)CCN1CCN(CCO)CC1</smiles>

Hepes

Fig. 4.The structures of the Tris-base and Hepes buffers used in this study.

The percentage dissociation of these buffers can be determined from the HendersonHasselbalch relationship as:

$$
\begin{gathered}
\frac{\text { TrisH }^{+}}{\text {TrisH }^{+}+\text {Tris }}=\frac{1}{1+x} \\
\frac{\text { Hepes }^{-}}{{\text {Hepes }+ \text { Hepes }^{-}}^{-}}=\frac{x}{1+x}
\end{gathered}
$$

where $x=10^{p H-p K a}$.

There are also small quantities of disodium EDTA, which will be fully dissociated into a large anion and two sodium cations. As the initial $\mathrm{pH}$ is 8.0 , there is also a small population of $\mathrm{OH}^{-}$ ions. The dense structure of the AEM provides good selectivity for passage of the $\mathrm{OH}^{-}$ion, due to its smaller ionic volume and higher electrical conductivity [31-33] compared to the EDTA and Hepes anions. Because of this high counter-ion permselectivity, it is probable that $\mathrm{OH}^{-}$passing from the cathode chamber to $\mathrm{C} 2$ through the AEM causes the $\mathrm{pH}$ rise of the $\mathrm{C} 2$ solution. For the same reason, $\mathrm{OH}^{-}$in $\mathrm{C} 1$ permeating through the AEM restriction membrane to the anode causes the $\mathrm{pH}$ decline in $\mathrm{C} 1$.

Similarly, the small and mobile $\mathrm{H}^{+}$generated from buffer dissociation will transfer from the anode to $\mathrm{C} 1$ and from $\mathrm{C} 2$ to the cathode in the two-CEM configuration, causing the $\mathrm{pH}$ to 
change in the same direction. However, as the $\mathrm{H}^{+}$concentration is very low at $\mathrm{pH} 8$ and as the relatively small $\mathrm{Na}^{+}$cation from the EDTA is available at higher concentrations $(0.002 \mathrm{M}$ versus $\left.10^{-8} \mathrm{M}\right)$, the transport of $\mathrm{H}^{+}$through the CEMs is small and so the $\mathrm{pH}$ change of the two-CEM system is much smaller than the two-AEM system. The PAm restriction membrane used in this study has a MWCO of $5 \mathrm{kDa}$, which is much higher than the molecular weight of Hepes (MW 238.3) and EDTA (MW 372.24), so this membrane does not exhibit any selectivity between ions and $\mathrm{pH}$ of the solutions is stable. A change of $\mathrm{pH}$ during the EDFM process only occurs in configurations where an IEM is used as the restriction membrane, because of their selectivity to ions of different size.

Although the $\mathrm{pH}$ change in the compartments caused by the selective permeation of $\mathrm{H}^{+}$and $\mathrm{OH}^{-}$is not significant (less than $1.5 \mathrm{pH}$ unit in all the conditions tested), it still has two possible consequences in EDFM operation. One is the possible change of electrophoretic mobility of the proteins, peptides or amino acids in the mixture [34-36]. The other consequence of this $\mathrm{pH}$ change is the abnormal conductivity change of the solutions. In particular, this movement of $\mathrm{OH}^{-}$ions can explain the unusual conductivity changes in the $\mathrm{C} 1$ and $\mathrm{C} 2$ solution in the twoAEM systems (Fig. 2(b)), which showed a net loss of conductivity. As the $\mathrm{pH}$ in $\mathrm{C} 2$ increases, the dissociation of Hepes increases, but that of Tris decreases (Fig. 5). As Tris is present at twice the concentration as Hepes $(0.02 \mathrm{M}$ versus $0.01 \mathrm{M})$, and as the decrease in dissociation of this buffer is greater than the increase of dissociation of Hepes, the net effect is a decline in conductivity, even though the total proportion of all ions is increasing in this chamber. Conversely, in C1, the dissociation of Tris increases to $96 \%$, while that of Hepes declines to $13 \%$ (Fig. 5). In this case, the change in dissociation of Hepes is greater than that of Tris. 
Coupling these changes to the fact that the total proportion of all ions is declining in this chamber means that the overall effects is again a decline in conductivity (Fig. 2).

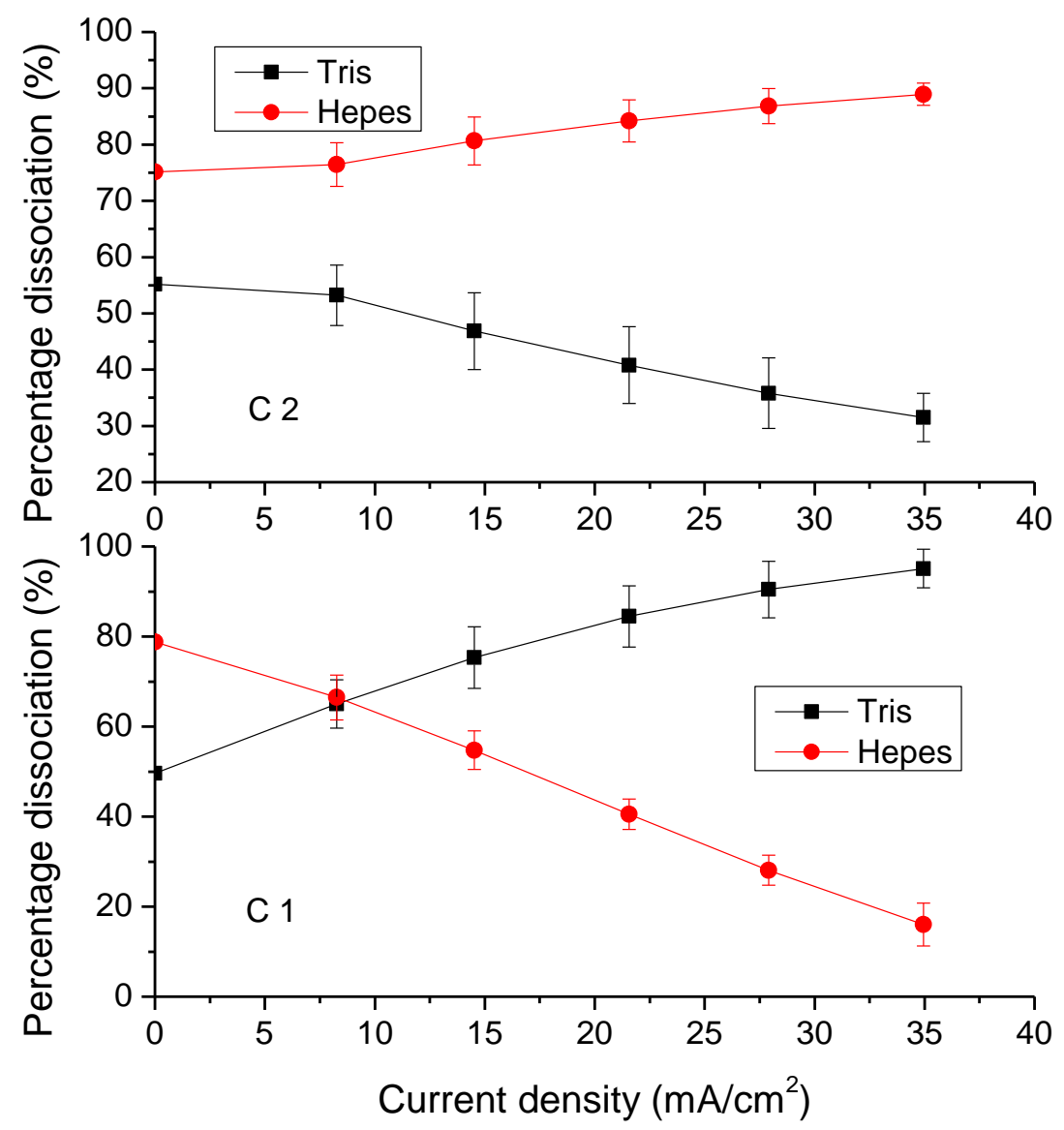

Fig.5. Percentage dissociation of Tris and Hepes in $\mathrm{C} 2$ and $\mathrm{C} 1$ in continuous processing with the twoAEM configuration shown in Fig. 1(c). The voltage varies from 0 to $210 \mathrm{~V}$. Error bars are based on replicate experiments.

Batch experiments with the three stable configurations were also conducted under the same conditions. The conductivity in the two inner compartments of the two-PAm and two-CEM configurations also showed a continuous change over time because of the transport number difference between the separation membrane and the restriction membranes (Fig. 6(a)). The conductivity of the solution in $\mathrm{C} 1$ of the two-AEM configuration shows an obvious decline 
with time, which indicates that the direction of the salt transfer is opposite to that of the other two configurations and is again consistent with the calculated result from Equations 7 and 8. However, the conductivity in C2 shows a seemingly random variation over time with the average conductivity only slightly higher than the original value (Fig. 6(b)).

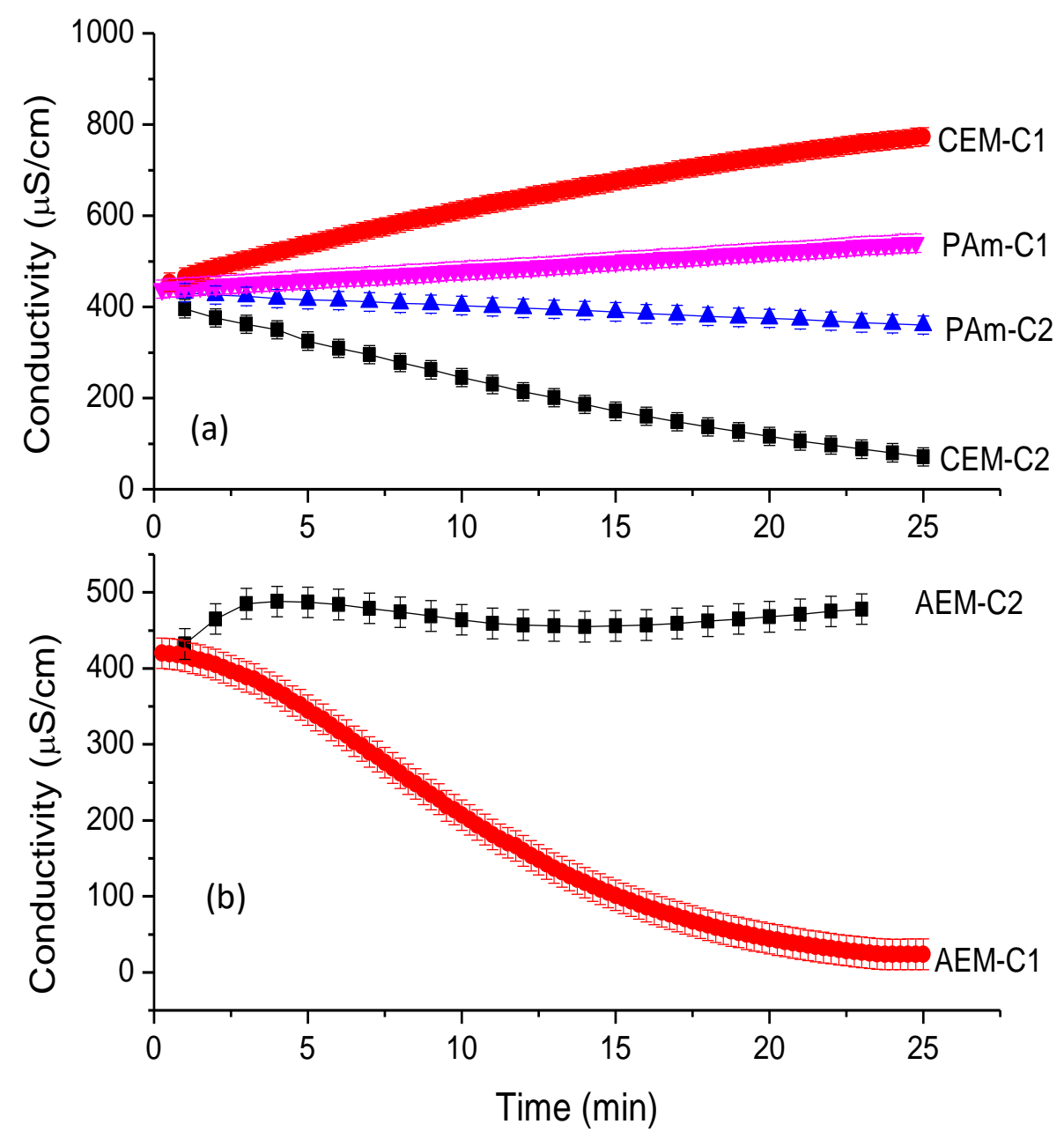

Fig. 6. Conductivity change in compartments 1 and 2 over time in batch experiments in (a) configurations with PAm and CEM as the restriction membrane and (b) configuration with AEM as the restriction membrane at $100 \mathrm{~V}$. Error bars are based on the stated accuracy of the conductivity meter.

The variation of $\mathrm{pH}$ as well as percentage dissociation of Tris and Hepes with time in $\mathrm{C} 2$ of the two-AEM system is shown in Fig.7. At the beginning of the experiment, the $\mathrm{pH}$ in $\mathrm{C} 2$ is 
relatively stable because of the buffer effect of the solution. A rise in conductivity is observed as the ion concentration increases during this period (Fig. 6(b)). Then the $\mathrm{pH}$ shows an obvious increase, which in turn reduces the percentage dissociation of Tris, and thus reduces the conductivity of the solution. The percentage dissociation of Tris declines to less than $20 \%$, while that of Hepes reaches $94 \%$ over this period of time. After 15 minutes of operation, the $\mathrm{pH}$ of the solution and the percentage dissociation of the buffers reach a plateau and the conductivity increases again with the increasing concentration of ions in this chamber.

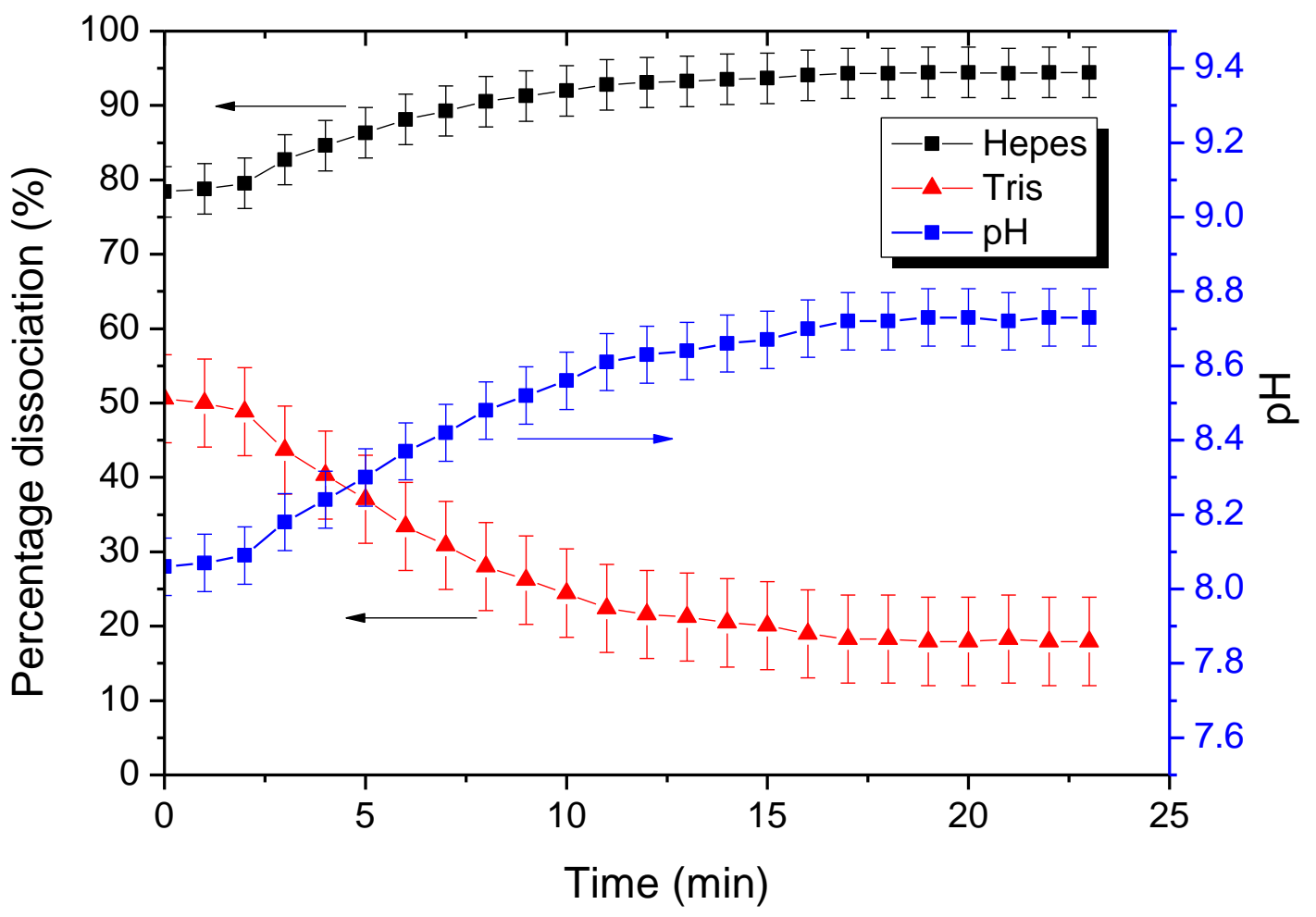

Fig.7. The change in $\mathrm{pH}$ and percentage dissociation of the two buffer species over time in $\mathrm{C} 2$ of the two-AEM system at $100 \mathrm{~V}$. Error bars are based on the stated accuracy of the pH probe. 


\subsection{Protein transfer}

The selectivity and flux of proteins is mainly determined by the characteristics of the central separation membrane within the EDFM setup (Fig. 1). However, the properties of the related restriction membranes also have an important effect on the transfer flux of proteins. Fig.8 shows the transfer of BSA as a function of the operating current density in a continuous EDFM process. The two-CEM system provides the highest BSA transfer, while the two-AEM system has the lowest BSA permeability for this protein, even though the feed solution of the twoAEM system shows the highest $\mathrm{pH}$ (Fig. 3), which in turn increases the negative charge density of BSA [37]. The transfer with the PAm membrane as restriction membranes is intermediate between these two other systems. This data illustrate the importance of the choice of restriction membrane. As the same separation membrane is used in all cases, the performance difference is caused by these restriction membranes and can be explained again by Equations 9 and 10.

In an EDFM system, charged proteins permeate through the separation membrane selectively driven by electrical potential. In the Tris-Hepes buffer with a $\mathrm{pH}$ of 8.0, BSA has a net negative charge and thus transfers from C2 to $\mathrm{C} 1$ in all the configurations shown in Fig. 1 (a), (b) and (c). As discussed in section 4.1, C1 has a higher total ion concentration than C2 in a two-CEM configuration, which means that the Fickian diffusion of anions is in the opposite direction to the electric potential gradient. Hence the overall anion permeability is reduced (Equation 10). Conversely for the configuration with AEMs as restriction membranes, the Fickian diffusion of anions is in the same direction as the potential gradient. Furthermore, the two-AEM configuration has greater anion permeability because of the larger concentration driving force for Fickian diffusion compared to the two-Pam membrane system. Since the total flux 
of negatively charged protein and anions is determined by the operating current and the characteristics of the separation membrane, the highest protein flux occurs for the two-CEM system, as calculated by Equation 9.

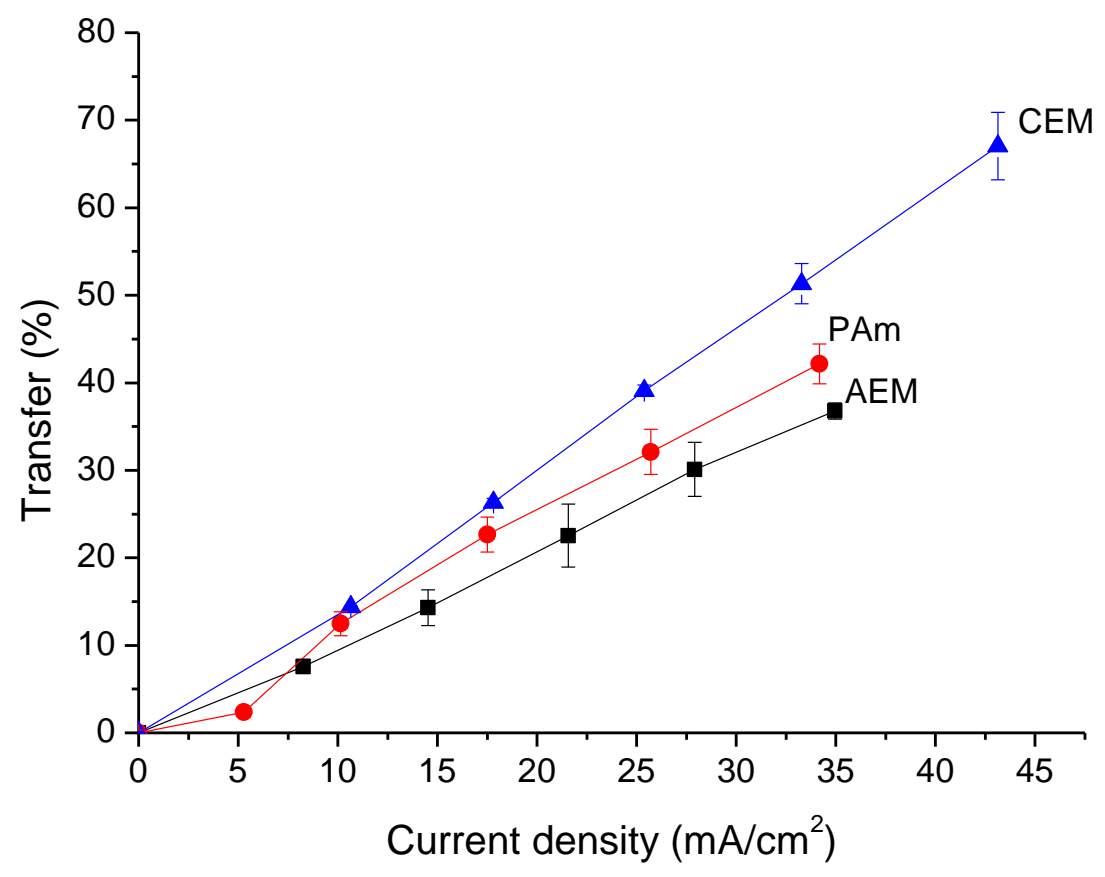

Fig. 8. The transfer performance of a model BSA protein with different restriction membranes (CEM, AEM and PAm membrane) in a continuous EDFM process. The voltage varies from 0 to $210 \mathrm{~V}$. Error bars are based on replicate experiments.

The effect of the restriction membrane type on the BSA transfer after 25 minutes of operation at $100 \mathrm{~V}$ in batch operation is shown in Fig. 9. The BSA transfer of the configuration with two CEMs as restriction membranes reaches nearly $90 \%$, while using PAm membrane and AEMs as restriction membranes can only result in less than $60 \%$ and less than $30 \%$ of BSA transfer, respectively. Because the different restriction membrane system also provides a variation in the current density (Fig. 10) as well as the migration direction of ions, the difference of BSA transfer between these three configurations is more significant than that in continuous processing at the same current density. It is also possible that some BSA precipitated onto the 
positively charged surface of the AEMs and was not recovered, while the protein was repelled from the negatively charged surface of the CEMs, reducing any membrane fouling. It should be noted that at the end of the experiment, the $\mathrm{pH}$ of the feed solution with CEM, PAm and AEM as restriction membrane was $8.64,8.18$ and 8.73 , respectively, which means that the net charge of BSA remained negative during these experiments.

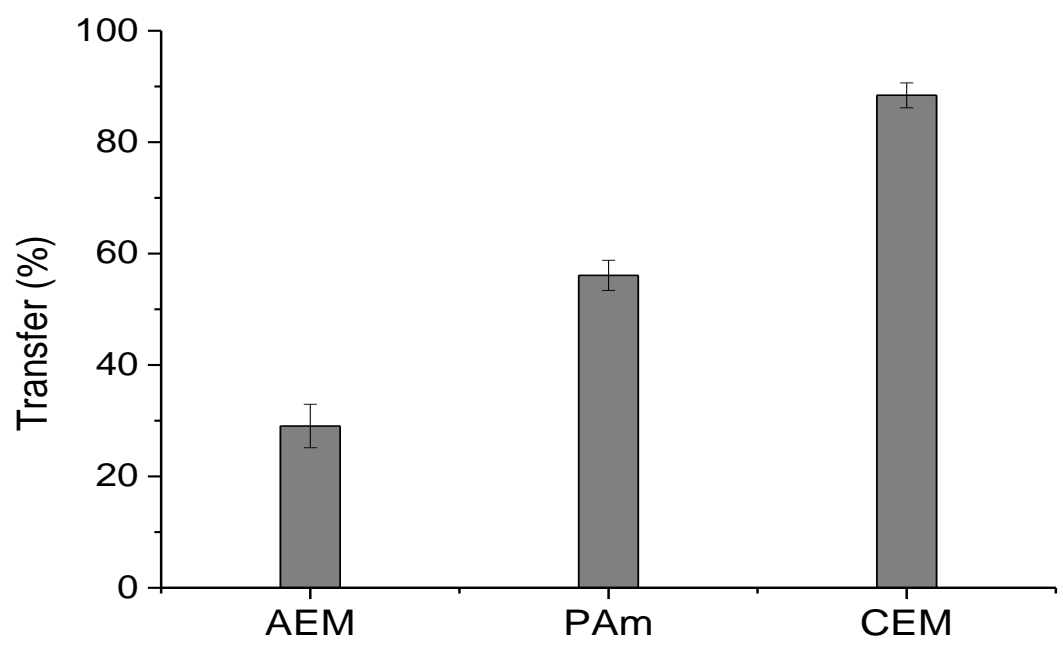

Fig. 9. The transfer of a model protein BSA with different restriction membranes (CEM, AEM and PAm membrane) in a batch EDFM process at 100V. Error bars are based on replicate experiments. 


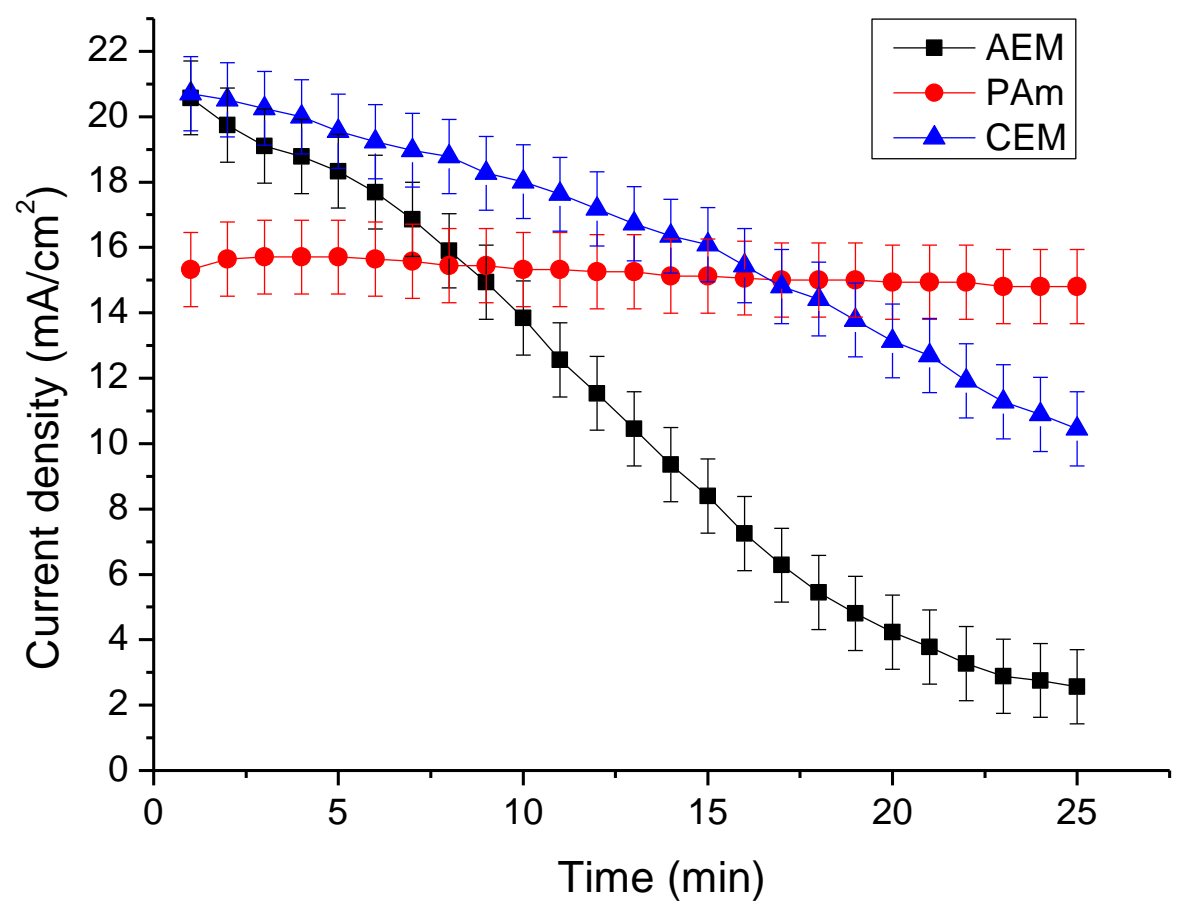

Fig. 10. Current density changes over time in batch experiments with different restriction membranes at 100V. Error bars are based on the stated accuracy of the power supply.

If the protein to be separated has a positive charge at the $\mathrm{pH}$ value of the buffer, it will transport through the separation membrane in the opposite direction (C1 to $\mathrm{C} 2$ ), together with the cations in the buffer. Since the concentration gradient is determined by the type of restriction membranes, the configuration with two AEMs as restriction membrane is expected to have the highest protein flux in this case. The configuration with the highest protein transfer flux is always preferred because it ensures a high current efficiency in the EDFM process.

\section{Conclusions}

Restriction membranes used in the EDFM process have the dual function of conducting current and restricting the leakage of proteins that are being separated. Either crosslinked 
non-charged polymer membranes such as polyacrylamide (PAm) or ion exchange membranes (IEMs) can be used for this purpose. In the present work, we have shown that the configuration with non-charged PAm membranes as restriction membranes exhibits the most stable operation during both batch and continuous operation, with only slight changes in concentration and a relatively stable $\mathrm{pH}$. In contrast, when IEMs are used, there are transport differences between restriction membranes and the separation membrane, leading to a concentration changes in the feed and permeate compartment. The solution in the compartment next to the anode is concentrated when two CEMs are used as restriction membranes, while that nearest to the cathode is concentrated when two-AEMs are used. Further, the selectivity of both IEMs for the smaller $\mathrm{H}^{+}$and $\mathrm{OH}^{-}$ions results in a $\mathrm{pH}$ change. With the Tris-Hepes buffer system used, the $\mathrm{pH}$ value in the compartment next to the cathode increases while that in the compartment next to the anode decreases in both AEM and CEM configurations. These $\mathrm{pH}$ changes in turn alter the extent of buffer dissociation and this leads to complicated changes in solution conductivity. The concentration changes in the two compartments also result in different rates of protein transfer. For negatively charged proteins, the two-CEM system shows the highest flux and we conclude that for positively charged proteins, AEM should provide the best choice for restriction membranes within the EDFM unit.

\section{Acknowledgements}

The authors thank Memphasys for their kind donation of both membranes and the EDFM cell. Dr. Huining Deng acknowledges funding from the China Scholarship Council. 


\begin{tabular}{|lll|}
\hline Nomenclature & & \\
C & concentration & \\
D & Fickian diffusion coefficient & $\mathrm{cm}^{2} / \mathrm{s}$ \\
F & Faraday constant & $96,485 \mathrm{~s} \mathrm{~A} / \mathrm{mol}$ \\
I & Current density & $\mathrm{A} / \mathrm{cm}^{2}$ \\
J & Flux & $\mathrm{mol} / \mathrm{cm}^{2} . \mathrm{s}$ \\
$\mathrm{m}$ & Mass of the solution & \\
T & lon transport number & \\
X & Distance across the membrane & $\mathrm{m}$ \\
z & Valence & \\
$\varphi$ & Electrical potential & \\
& & \\
Subscripts & & \\
A & & \\
A' & Anion & \\
C & All anions except the proteins & \\
f & Cation & \\
i & Feed & \\
p & Each ionic species & \\
SM & permeate & \\
\hline
\end{tabular}

\section{References}

[1] C. Roblet, M.J. Akhtar, S. Mikhaylin, G. Pilon, T. Gill, A. Marette, L. Bazinet, Enhancement of glucose uptake in muscular cell by peptide fractions separated by electrodialysis with filtration membrane from salmon frame protein hydrolysate, Journal of Functional Foods, 22 (2016) 337-346.

[2] H. Lu, W. Zou, P. Chai, J. Wang, L. Bazinet, Feasibility of antibiotic and sulfate ions separation from wastewater using electrodialysis with ultrafiltration membrane, J. Clean, Prod., 112 (2016) 3097-3105. [3] S. Suwal, C. Roblet, J. Amiot, L. Bazinet, Presence of free amino acids in protein hydrolysate during electroseparation of peptides: Impact on system efficiency and membrane physicochemical properties, Sep. Purif. Technol., 147 (2015) 227-236.

[4] S. Suwal, C. Roblet, J. Amiot, A. Doyen, L. Beaulieu, J. Legault, L. Bazinet, Recovery of valuable peptides from marine protein hydrolysate by electrodialysis with ultrafiltration membrane: impact of ionic strength, Food Res. Int., 65 (2014) 407-415.

[5] S. Galier, H. Roux-de Balmann, Study of the mass transfer phenomena involved in an electrophoretic membrane contactor, J. Membr, Sci., 194 (2001) 117-133.

[6] S. Galier, H. Roux-de Balmann, Influence of electrostatic interactions in electrophoretic membrane contactors, Desalination, 149 (2002) 351-356.

[7] S. Galier, H. Roux-de Balmann, The electrophoretic membrane contactor: A mass-transfer-based methodology applied to the separation of whey proteins, Sep. Purif. Technol., 77 (2011) 237-244.

[8] S. Galier, H. Balmann, Study of biomolecules separation in an electrophoretic membrane contactor, J. Membr, Sci., 241 (2004) 79-87.

[9] D. Ogle, M. Sheehan, B. Rumbel, T. Gibson, D.B. Rylatt, Design of a new, twelve-channel electrophoretic apparatus based on the Gradiflow technology, J. Chromatogr. A, 989 (2003) 65-72. 
[10] R. He, A.T. Girgih, E. Rozoy, L. Bazinet, X.-R. Ju, R.E. Aluko, Selective separation and concentration of antihypertensive peptides from rapeseed protein hydrolysate by electrodialysis with ultrafiltration membranes, Food Chem., 197 (2016) 1008-1014.

[11] A. Doyen, C. Roblet, L. Beaulieu, L. Saucier, Y. Pouliot, L. Bazinet, Impact of water splitting phenomenon during electrodialysis with ultrafiltration membranes on peptide selectivity and migration, J. Membr, Sci., 428 (2013) 349-356.

[12] A. Doyen, L. Beaulieu, L. Saucier, Y. Pouliot, L. Bazinet, Demonstration of in vitro anticancer properties of peptide fractions from a snow crab by-products hydrolysate after separation by electrodialysis with ultrafiltration membranes, Sep. Purif. Technol., 78 (2011) 321-329.

[13] E. Serre, E. Rozoy, K. Pedneault, S. Lacour, L. Bazinet, Deacidification of cranberry juice by electrodialysis: Impact of membrane types and configurations on acid migration and juice physicochemical characteristics, Sep. Purif. Technol., 163 (2016) 228-237.

[14] S. Suwal, J. Amiot, L. Beaulieu, L. Bazinet, Effect of pulsed electric field and polarity reversal on peptide amino acid migration, selectivity and fouling mitigation, J. Membr, Sci., 510 (2016) 405-416.

[15] S. Mikhaylin, V. Nikonenko, G. Pourcelly, L. Bazinet, Hybrid bipolar membrane electrodialysis/ultrafiltration technology assisted by a pulsed electric field for casein production, Green Chem., 18 (2016) 307-314.

[16] S. Mikhaylin, V. Nikonenko, G. Pourcelly, L. Bazinet, Intensification of demineralization process and decrease in scaling by application of pulsed electric field with short pulse/pause conditions, J. Membr, Sci., 468 (2014) 389-399.

[17] C. Roblet, A. Doyen, J. Amiot, L. Bazinet, Impact of pH on ultrafiltration membrane selectivity during electrodialysis with ultrafiltration membrane (EDUF) purification of soy peptides from a complex matrix, J. Membr, Sci., 435 (2013) 207-217.

[18] A. Doyen, L. Beaulieu, L. Saucier, Y. Pouliot, L. Bazinet, Impact of ultrafiltration membrane material on peptide separation from a snow crab byproduct hydrolysate by electrodialysis with ultrafiltration membranes, J. Agric. Food. Chem., 59 (2011) 1784-1792.

[19] A. Saxena, V.K. Shahi, pH controlled selective transport of proteins through charged ultrafilter membranes under coupled driving forces: An efficient process for protein separation, J. Membr, Sci., 299 (2007) 211-221.

[20] Z.S. Horvath, G.L. Corthals, C.W. Wrigley, J. Margolis, Multifunctional apparatus for electrokinetic processing of proteins, Electrophoresis, 15 (1994) 968-971.

[21] R.K. Nagarale, G.S. Gohil, V.K. Shahi, Recent developments on ion-exchange membranes and electro-membrane processes, Adv. Colloid Interface Sci., 119 (2006) 97-130.

[22] G. Chen, W. Song, B. Qi, J. Li, R. Ghosh, Y. Wan, Separation of protein mixtures by an integrated electro-ultrafiltration-electrodialysis process, Sep. Purif. Technol., 147 (2015) 32-43.

[23] S. Suwal, C. Roblet, A. Doyen, J. Amiot, L. Beaulieu, J. Legault, L. Bazinet, Electrodialytic separation of peptides from snow crab by-product hydrolysate: Effect of cell configuration on peptide selectivity and local electric field, Sep. Purif. Technol., 127 (2014) 29-38.

[24] H. Miyoshi, Diffusion coefficients of ions through ion-exchange membranes for Donnan dialysis using ions of the same valence, Chem. Eng. Sci., 52 (1997) 1087-1096.

[25] D.B. Rylatt, M. Napoli, D. Ogle, A. Gilbert, S. Lim, C.H. Nair, Electrophoretic transfer of proteins across polyacrylamide membranes, J. Chromatogr. A, 865 (1999) 145-153.

[26] X.-D. Wang, X.-Q. Chen, H.-H. Yang, G.-Y. Hu, Comparison of the effects of cholinesterase inhibitors on [3 H] MK-801 binding in rat cerebral cortex, Neurosci. Lett., 272 (1999) 21-24.

[27] H. He, M.A. Mortellaro, M.J. Leiner, R.J. Fraatz, J.K. Tusa, A fluorescent sensor with high selectivity and sensitivity for potassium in water, J. Amer. Chem. Soc., 125 (2003) 1468-1469.

[28] C. Gedeon, G. Anger, M. Piquette-Miller, G. Koren, Breast cancer resistance protein: mediating the trans-placental transfer of glyburide across the human placenta, Placenta, 29 (2008) 39-43.

[29] G. Chen, W. Song, B. Qi, R. Ghosh, Y. Wan, Separation of human serum albumin and polyethylene glycol by electro-ultrafiltration, Biochem. Eng. J., 109 (2016) 127-136. 
[30] J.-F. Poulin, J. Amiot, L. Bazinet, Simultaneous separation of acid and basic bioactive peptides by electrodialysis with ultrafiltration membrane, J. Biotechnol., 123 (2006) 314-328.

[31] H. Miyoshi, Diffusion coefficients of ions through ion exchange membrane in Donnan dialysis using ions of different valence, J. Membr, Sci., 141 (1998) 101-110.

[32] B. Van der Bruggen, A. Koninckx, C. Vandecasteele, Separation of monovalent and divalent ions from aqueous solution by electrodialysis and nanofiltration, Water Res., 38 (2004) 1347-1353.

[33] L. Bazinet, D. Ippersiel, C. Gendron, J. Beaudry, B. Mahdavi, J. Amiot, F. Lamarche, Cationic balance in skim milk during bipolar membrane electroacidification, J. Membr, Sci., 173 (2000) 201-209.

[34] N. Ndiaye, Y. Pouliot, L. Saucier, L. Beaulieu, L. Bazinet, Electroseparation of bovine lactoferrin from model and whey solutions, Sep. Purif. Technol., 74 (2010) 93-99.

[35] S. Saksena, A.L. Zydney, Effect of solution $\mathrm{pH}$ and ionic strength on the separation of albumin from immunoglobulins (IgG) by selective filtration, Biotechnol. Bioeng., 43 (1994) 960-968.

[36] O. Kattan-Readi, K. Wiratha, D. Nijmeijer, On the isolation of single basic amino acids with electrodialysis for the production of biochemicals, Ind. Eng. Chem. Res., 52 (2013) 1069-1078.

[37] J. Ma, L. Qin, X. Zhang, H. Huang, Temporal evolution of the selectivity-permeability relationship during porous membrane filtration of protein solutions, J. Membr, Sci., 514 (2016) 385-397. 